PROCEEDINGS OF THE

AMERICAN MATHEMATICAL SOCIETY

Volume 127, Number 10, Pages 3035-3041

S 0002-9939(99)04893-5

Article electronically published on April 23, 1999

\title{
VOLUME GROWTH AND HOLONOMY IN NONNEGATIVE CURVATURE
}

\author{
KRISTOPHER TAPP
}

(Communicated by Christopher Croke)

\begin{abstract}
The volume growth of an open manifold of nonnegative sectional curvature is proven to be bounded above by the difference between the codimension of the soul and the maximal dimension of an orbit of the action of the normal holonomy group of the soul. Additionally, an example of a simplyconnected soul with a non-compact normal holonomy group is constructed.
\end{abstract}

\section{InTRODUCTION}

For a Riemannian manifold $M^{n}$ with nonnegative sectional curvature, the volume of a ball $B_{\Sigma}(r)$ of radius $r$ about a compact totally geodesic submanifold $\Sigma^{n-k}$ can be estimated by a generalized Bishop-Gromov type inequality which states that

$$
V(r):=\frac{\operatorname{Vol}\left(B_{\Sigma}(r)\right)}{r^{k}}
$$

is a monotonically nonincreasing function of $r$ [3]. If $M$ is open, this implies that the volume growth of $M$ is not greater than the codimension $k$ of $\Sigma$, where the volume growth, $\operatorname{VG}(M)$, of $M$ is defined as follows:

$$
\operatorname{VG}(M)=\inf \left\{x \in \mathbb{R} \mid \lim _{r \rightarrow \infty} \frac{\operatorname{Vol}\left(B_{p}(r)\right)}{r^{x}}=0\right\} .
$$

Note that this definition is independent of the choice of $p \in M$.

The case where $\Sigma$ is a soul of $M$ (in the sense of [2]) and the volume growth of $M$ is maximal in the sense that $\operatorname{VG}(M)=k$ was studied by Schroeder and Strake in [7]. Their main result states that in this case the reduced holonomy group $\Phi^{0}$ of the normal bundle $\nu(\Sigma)$ of $\Sigma$ in $M$ is trivial. Hence, as they pointed out, if $M$ is additionally assumed to be simply connected, the main result of [9] implies that $M$ splits isometrically.

Our main theorem is the following generalization of Schroeder and Strake's result: $\mathrm{VG}(M) \leq k-m$, where $m$ denotes the maximal dimension of an orbit of the action of $\Phi^{0}$ on the fiber $\nu_{p}(\Sigma)$ of $\nu(\Sigma)$ at $p \in \Sigma$. Additionally, we prove the related fact that along almost every radial geodesic in $M$, there exist at least $m$ linearly independent bounded vertical Jacobi fields.

Received by the editors December 11, 1997.

1991 Mathematics Subject Classification. Primary 53C20; Secondary 57S20.

Key words and phrases. Volume growth, holonomy, nonnegative curvature, soul.

(C)1999 American Mathematical Society 
The possibility that $\Phi^{0}$ might be a non-closed subgroup of $\mathrm{SO}(k)$ introduces certain technical difficulties in our proofs. In the final section, we justify these worries by exhibiting a soul with non-compact reduced normal holonomy.

The author would like to express his thanks to Professor Ziller and Professor Croke for their help in the preparation of this paper.

\section{BACKground: The Metric Projection onto the soul}

Perelman's main result in [6] is the primary tool which allows us to simplify and generalize the arguments of Schroeder and Strake. This result states that the metric projection $\pi: M \rightarrow \Sigma$, which maps the point $x \in M$ to the point $\pi(x)$ in $\Sigma$ to which it is closest, is well-defined and is in fact a $C^{1}$ Riemannian submersion.

There is a simple way to describe the horizontal distribution of $\pi$. Let $\exp ^{\perp}$ : $\nu(\Sigma) \rightarrow M$ denote the normal exponential map, and write $T(\nu(\Sigma))=\widetilde{\mathcal{H}} \oplus \widetilde{\mathcal{V}}$ for the decomposition of the tangent bundle of $\nu(\Sigma)$ into the horizontal and vertical spaces associated to the connection on the normal bundle. Similarly, write $T M=\mathcal{H} \oplus \mathcal{V}$ for the orthogonal decomposition of $T M$ into the horizontal and vertical distributions associated with the Riemannian submersion $\pi$. Then

$$
\mathcal{H}=d \exp ^{\perp}(\widetilde{\mathcal{H}}) .
$$

If $\alpha(t)$ is a path in $\Sigma$ from $p=\alpha(0)$ to $q=\alpha(1)$, and $x \in \pi^{-1}(p)$, then there is a simple description of the horizontal lift $\bar{\alpha}$ of $\alpha$ to $x$. Write $x=\exp ^{\perp}(v)$ for some $v \in \nu_{p}(\Sigma)$. Then

$$
\bar{\alpha}(t)=\exp ^{\perp}\left(P_{\left.\alpha\right|_{[0, t]}}(v)\right) .
$$

Here $P_{\left.\alpha\right|_{[0, t]}}(v)$ denotes the parallel transport of $v$ along the appropriate segment of $\alpha$. This path is clearly a lift of $\alpha$, and it is horizontal because it's preimage in $\nu(\Sigma)$ is horizontal.

It is worth noting that Perelman's theorem provides an elementary proof of the previously mentioned fact that $\operatorname{VG}(M) \leq k$. For, if $\nu(\Sigma)$ is endowed with the connection metric with flat fibers, then $\exp ^{\perp}$ becomes a distance non-increasing map. To see this, take any $v \in \nu(\Sigma)$ and let $x:=\exp ^{\perp}(v)$. The above considerations show that $d\left(\exp ^{\perp}\right)_{v}$ maps $\widetilde{\mathcal{H}}_{v}$ isometrically onto $\mathcal{H}_{x}$. Also, Rauch's theorem implies that $d\left(\exp ^{\perp}\right)_{v}$ maps $\widetilde{\mathcal{V}}_{v}$ in a distance non-increasing way into $\mathcal{V}_{x}$. Since $\operatorname{VG}(\nu(\Sigma))=$ $k$, it follows that $\operatorname{VG}(M) \leq k$.

\section{VOlume GROWTH AND HOLONOMY}

In this section, the main theorem is proven. Since $\Phi^{0}$ might be non-compact, most of the theory of transformation groups does not apply to the action of $\Phi^{0}$ on a fiber $\nu_{p}(\Sigma)$. We therefore require the following two lemmas about Lie subgroups of $\mathrm{SO}(k)$.

Lemma 3.1. Let $G$ be a (possibly non-compact) connected Lie subgroup of $S O(k)$. The union, $\Omega$, of all orbits of the action of $G$ on $S^{k-1}(1)$ which have maximal dimension is open and dense.

Proof. $\Omega$ is trivially open. To establish that $\Omega$ is dense, decompose the Lie algebra $\mathcal{G}$ of $G$ as $\mathcal{G}=\mathcal{Z} \oplus \mathcal{A}$, where $\mathcal{Z}$ is the center of $\mathcal{G}$, and $\mathcal{A}$ is semisimple. Let $Z$ and $A$ denote the Lie subgroups of $G$ associated with $\mathcal{Z}$ and $\mathcal{A}$, respectively. $Z$ is abelian 
and $A$ is compact. $Z$ is a subgroup of a maximal torus of $\mathrm{SO}(k)$, and with respect to a properly chosen basis of $\mathbb{R}^{k}, Z$ embeds in $\mathrm{SO}(k)$ as follows:

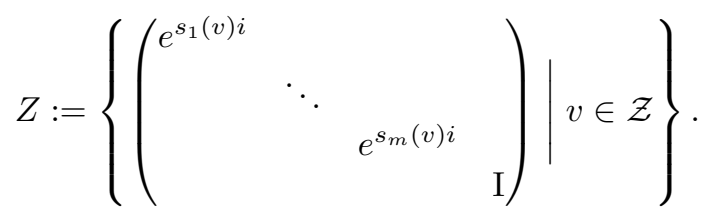

Here, each $s_{i}$ is a linear function from $\mathcal{Z}$ to $\mathbb{R}$. Write $\mathbb{R}^{k}=V_{1} \oplus \cdots \oplus V_{m} \oplus W$ for the corresponding orthogonal decomposition of $\mathbb{R}^{k} ; \operatorname{dim}\left(V_{i}\right)=2$ and $\operatorname{dim}(W)=k-2 m$.

For any $v \in S^{k-1}(1), \operatorname{dim}(G(v)) \leq \operatorname{dim}(Z(v))+\operatorname{dim}(A(v))$, and we claim that equality holds on an open dense subset $E$ of $S^{k-1}(1)$. To construct $E$, consider a basis $\left\{X_{1}, \ldots, X_{a}\right\}$ of $\mathcal{Z}$ and $\left\{Y_{1}, \ldots, Y_{b}\right\}$ of $\mathcal{A}$, and regard the elements $X_{i}$ and $Y_{i}$ as Killing vector fields on $\mathbb{R}^{k}$. For each $i=1, \ldots, b$, the set $K_{i}:=\left\{w \in W \mid Y_{i}(w)=0\right\}$ is a linear subspace of $W$, which is not necessarily proper. Define:

$$
E:=\left\{v_{1}+\cdots+v_{m}+w \in S^{k-1}(1) \mid \text { each } v_{i} \neq 0 \text { and if } w \in K_{i} \text {, then } K_{i}=W\right\} .
$$

$E$ is clearly open and dense. For every $v \in E$ and any $i=1, \ldots, b, Y_{i}(v) \notin$ $\operatorname{span}\left\{X_{1}(v), \ldots, X_{a}(v)\right\}$. If $i$ is such that $K_{i} \neq W$, this is clear from construction; otherwise, $Y_{i}(v) \in \operatorname{span}\left\{X_{1}(v), \ldots, X_{a}(v)\right\}$ implies $Y_{i} \in \mathcal{Z}$, which is impossible. Therefore, $\operatorname{dim}(G(v))=\operatorname{dim}(Z(v))+\operatorname{dim}(A(v))$ for every $v \in E$.

From the previous description of $Z$, one can easily see that the union, $\Omega_{Z}$, of all orbits of the action of $Z$ on $S^{k-1}(1)$ which have maximal dimension is open and dense in $S^{k-1}(1)$. Further, $\Omega_{A}$ (defined analogously) is open and dense by the theory of compact group actions. Therefore, $\Omega_{Z} \cap \Omega_{A} \cap E$ is an open dense subset of $S^{k-1}(1)$ which is contained in $\Omega$. It follows that $\Omega$ is dense.

Lemma 3.2. Let $G$ be a (possibly non-compact) Lie subgroup of $S O(k)$, and $C$ be a closed neighborhood of the identity in $G$. Let $m$ denote the maximal dimension of an orbit of the action of $G$ on $\mathbb{R}^{k}$. Then there exists a finite union, $W=\bigcup W_{i}$, of $(k-m)$-dimensional subspaces $W_{i}$ of $\mathbb{R}^{k}$ such that for any $u \in \mathbb{R}^{k}$, there exists some $g \in C$ with $g(u) \in W$.

Proof. The proof is by induction on $k$. The statement is trivial in dimensions $k=1$ or 2 . Assume that the lemma is true in dimensions less than $k$. Let $C \subset$ $G \subset \mathrm{SO}(k)$, as in the statement of the lemma. For every $v \in S^{k-1}(1) \subset \mathbb{R}^{k}$, we will construct an open neighborhood $U$ of $v$ in $S^{k-1}(1)$ and a finite union, $W=\bigcup W_{i}$, of $(k-m)$-dimensional subspaces $W_{i}$ of $\mathbb{R}^{k}$ which contain $v$, such that for every $u \in U$, there exists some $g \in C$ with $g(u) \in W$. Since $S^{k-1}(1)$ is compact, this will complete the proof.

Fix $v \in S^{k-1}(1)$. Let $m_{v}$ denote the dimension of the orbit $G(v)$. Decompose the Lie algebra $\mathcal{G}$ of $G$ as $\mathcal{G}=\mathcal{G}_{v} \oplus \operatorname{span}\left\{X_{1}, \ldots, X_{m_{v}}\right\}$, where $\mathcal{G}_{v}$ denotes the Lie algebra of the stabilizer $G_{v}$, and the elements $X_{i}$, when considered as Killing vector fields on $S^{k-1}(1)$, form a basis of $T_{v} G(v)$ at $v$. Define $Y$ to be the orthogonal compliment of $T_{v} G(v)$ in $T_{v} \mathbb{R}^{k} \approx \mathbb{R}^{k}$. $Y$ is a $\left(k-m_{v}\right)$-dimensional subspace of $\mathbb{R}^{k}$ containing $v$, and we claim that the set $\left\{g(u) \mid g \in C, u \in S^{k-1}(1) \cap Y\right\}$ contains an open neighborhood $U$ of $v$ in $S^{k-1}(1)$. This follows from the fact that the derivative at (id, $v$ ) of the map $\Psi: G \times\left(Y \cap S^{k-1}(1)\right) \rightarrow S^{k-1}(1)$ defined as $\Psi(g, u):=g(u)$ is surjective.

If the orbit $G(v)$ has maximal dimension, that is if $m_{v}=m$, then the single subspace $W:=Y$ and the open set $U$ will be as required for the construction. 
Otherwise, the dimension of $Y\left(=k-m_{v}\right)$ is too large, so $Y$ cannot serve as the required subspace. In this case, we will choose $W$ to be a union of smaller subspaces of $Y$, as follows.

The stabilizer $G_{v}$ acts by isometries on the subspace $Y^{\prime}$ consisting of those vectors in $Y$ which are orthogonal to $v$; we claim that the maximal dimension of an orbit of this action is $m-m_{v}$. To see this, choose a neighborhood $E$ of $v$ in $Y \cap S^{k-1}(1)$ small enough so that $\left\{X_{i}(w)\right\}$ is linearly independent and transverse to $Y$ for all $w \in E$. Then, for any $w \in E, G_{v}(w) \subset Y \cap S^{k-1}(1)$, and $T_{w} G(w)=T_{w} G_{v}(w) \oplus$ $\operatorname{span}\left\{X_{i}(w)\right\}$. Therefore, $\operatorname{dim}(G(w))=\operatorname{dim}\left(G_{v}(w)\right)+m_{v}$. From Lemma 3.1 and the fact that $G(E)$ contains an open neighborhood of $v$ in $S^{k-1}(1)$, it follows that $E$ must contain a vector $w$ for which $\operatorname{dim}(G(w))=m$. For this $w, \operatorname{dim}\left(G_{v}(w)\right)=$ $m-m_{v}=\max \left\{\operatorname{dim}\left(G_{v}\left(w^{\prime}\right)\right) \mid w^{\prime} \in Y^{\prime}\right\}$.

By the inductive hypothesis, there exists a finite union, $V:=\bigcup V_{i}$, of $(k-m-1)$ dimensional subspaces $V_{i}$ of $Y^{\prime}$ such that for any $u \in Y^{\prime}$ there exist an element $g \in G_{v} \cap C$ such that $g(u) \in V$. Define $W_{i}:=\operatorname{span}\left\{v, V_{i}\right\}$, and $W:=\bigcup W_{i}$. Each subspace $W_{i}$ has dimension $k-m$, and by construction, $\left\{g(u) \mid g \in G_{v} \cap C, u \in\right.$ $W\}=Y$. It follows that the set $\left\{g(u) \mid g \in C, u \in S^{k-1}(1) \cap W\right\}$ will contain the open neighborhood $U$ of $v$ defined above. This completes the proof.

Main Theorem. $\operatorname{VG}(M) \leq k-m$.

Proof. Fix $p \in \Sigma$. The proof turns on a modification of [7, prop. 1], which states that if $\Phi^{0}$ is compact, then all of $\Phi^{0}$ can be represented using loops at $p$ in $\Sigma$ of bounded length. Even when the compactness assumption is dropped, the proof of [7, prop. 1] still establishes the following: there exist a constant $b_{1}$ and a closed neighborhood $C$ of the identity in $\Phi^{0}$ such that any $g \in C$ can be represented as parallel translation along a loop at $p$ in $\Sigma$ with length $\leq b_{1}$. By Lemma 3.2, we can then find a finite union, $W:=\bigcup W_{i}$, of $(k-m)$-dimensional subspaces $W_{i}$ of $\nu_{p}(\Sigma)$ which meets every orbit of $C$ in the sense of the lemma.

Next we argue that all of $M$ is contained in a ball of radius $b_{2}:=b_{1}+\operatorname{diam}(\Sigma)$ about $\exp ^{\perp}(W)$. To see this, take any point $x \in M$, and write $x=\exp ^{\perp}(v)$ for some $v \in \nu(\Sigma)$. There exists a path $\alpha$ in $\Sigma$ of length less than $b_{2}$ such that $P_{\alpha}(v) \in W$ (namely, take any minimal path from $q:=\pi(v)$ to $p$, followed by a loop at $p$ which represents the proper holonomy element). The horizontal lift $\bar{\alpha}$ of $\alpha$ to $x$ is then a path in $M$ of the same length connecting $x$ to $y:=\exp ^{\perp}\left(P_{\alpha}(v)\right) \in \exp ^{\perp}(W)$. Therefore $B_{\exp ^{\perp}(W)}\left(b_{2}\right)=M$. Additionally, observe that if $v$ was chosen so that the radial geodesic $t \mapsto \exp ^{\perp}(t v)$ provides a minimal connection from $q$ to $x$, it follows that the radial geodesic $t \mapsto \exp ^{\perp}\left(t P_{\alpha}(v)\right)$ will provide a minimal connection from $p$ to $y$.

We conclude with an argument similar to the proof of [7, Thm. 2], by showing that there is a constant $K$ such that for large real numbers $r>0$,

$$
\operatorname{Vol}\left(B_{\Sigma}(r)-B_{\Sigma}(r-1)\right) \leq K r^{k-m-1} .
$$

This clearly implies the statement of the theorem.

Let $W^{1}$ be the set of unit-length vectors in $W$. Let $r$ be a positive real number. Take a net of points $\left\{v_{i}\right\}$ in $W^{1}$ such that every vector in $W^{1}$ makes an angle of less than $1 / r$ with some $v_{i}$. Since $W^{1}$ is a finite union of round spheres of dimension $k-m-1$, we need no more than $b_{3} r^{k-m-1}$ points $v_{i}$, where $b_{3}$ is a constant which does not depend on $r$. 
Now we claim that

$$
B_{\Sigma}(r)-B_{\Sigma}(r-1) \subset \bigcup_{i} B_{\exp ^{\perp}\left(r v_{i}\right)}\left(b_{2}+2\right) .
$$

To see this, take any point $x \in M$ with $r-1 \leq \operatorname{dist}(x, \Sigma) \leq r$. As described above, $x$ has distance $\leq b_{2}$ from some point $y=\exp ^{\perp}(v)$, where $v \in W$, and $\gamma(t):=\exp ^{\perp}(t v)$ provides a minimal connection between $p$ and $y$. Let $v_{i}$ be a point of the net which makes an angle less than $1 / r$ with $v$. Since $\gamma$ is minimizing, we can apply Toponogov to conclude that $d\left(y, \exp ^{\perp}\left(r v_{i}\right)\right) \leq 2$. Thus $\operatorname{dist}\left(x, \exp ^{\perp}\left(r v_{i}\right)\right) \leq b_{2}+2$.

Finally, since the Bishop-Gromov inequality implies that the volume of each ball $B_{\exp ^{\perp}\left(t v_{i}\right)}\left(b_{2}+2\right)$ is not greater than the volume $b_{4}$ of a ball of the same radius in the Euclidean space of the same dimension, we have

$$
\operatorname{Vol}\left(B_{\Sigma}(r)-B_{\Sigma}(r-1)\right) \leq b_{4} b_{3} r^{k-m-1} .
$$

So choosing $K:=b_{4} b_{3}$ is as required to conclude the proof.

\section{HolONOMY AND JaCOBI FIELDS}

This section establishes the following relationship between the amount of holonomy and the number of bounded vertical Jacobi fields along radial geodesics (i.e., geodesics which intersect the soul orthogonally):

Proposition 4.1. Along almost every radial geodesic in $M$, there exist at least $m$ linearly independent bounded vertical Jacobi fields.

Proof. Begin by choosing a collection $\left\{\tau_{1}, \ldots, \tau_{a}\right\}$ of paths in $\Sigma$ beginning at $p$, and collections $\left\{X_{1}, \ldots, X_{a}\right\}$, and $\left\{Y_{1}, \ldots, Y_{a}\right\}$ of vectors in $T_{p} \Sigma$ such that the set $\left\{V_{i}:=P_{\tau_{i}}^{-1} \circ R\left(P_{\tau_{i}}\left(X_{i}\right), P_{\tau_{i}}\left(Y_{i}\right)\right) \circ P_{\tau_{i}}\right\}$ is a basis of the Lie algebra of $\Phi^{0}$. This is possible by a theorem of Ambrose and Singer; see for example [5, Thm. 8.1]. Consider each $V_{i}$ to be a vector field on the unit normal sphere $\nu_{p}^{1}(\Sigma)$. Let $\Omega$ be the union of all orbits of the action of $\Phi^{0}$ on $\nu_{p}^{1}(\Sigma)$ which have maximal dimension. By Lemma 3.1, $\Omega$ has full measure. Fix $w \in \Omega$. $\left\{V_{i}(w)\right\}$ spans an $m$-dimensional subspace of $T_{w}\left(\nu_{p}^{1}(\Sigma)\right)$. To simplify notation, re-index so that $\left\{V_{1}(w), \ldots, V_{m}(w)\right\}$ is linearly independent. For each $i$ between 1 and $m$, consider the Jacobi field $J_{i}(t)$ along $\gamma(t):=\exp ^{\perp}(t w)$ with initial conditions $J_{i}(0)=0$ and $J_{i}^{\prime}(0)=V_{i}(w)$. We claim that each $J_{i}$ is a bounded vertical Jacobi field.

To see this, let $\tilde{J}_{i}(t)$ be the Jacobi field along $\tilde{\gamma}_{i}(t):=\exp ^{\perp}\left(t P_{\tau_{i}}(w)\right)$ with initial conditions $\tilde{J}_{i}(0)=0$ and $\tilde{J}_{i}^{\prime}(0)=P_{\tau_{i}}\left(V_{i}(w)\right)$. We first claim that $\tilde{J}_{i}$ is a bounded vertical Jacobi field. To see this, let $\tilde{X}_{i}(t)$, and $\tilde{Y}_{i}(t)$ be the lifts of $\tilde{X}_{i}(0):=$ $P_{\tau_{i}}\left(X_{i}\right)$ and $\tilde{Y}_{i}(0):=P_{\tau_{i}}\left(Y_{i}\right)$ to horizontal vector fields along $\tilde{\gamma}_{i}(t)$. Then, $\tilde{J}_{i}(t)=$ $-2 A\left(\tilde{X}_{i}(t), \tilde{Y}_{i}(t)\right)$, where $A$ denotes the $A$-tensor of $\pi$; see for example [8, Prop. 1.7]. Thus $\tilde{J}_{i}$ is vertical, and bounded because the $A$-tensor is bounded on $M$ by O'Neill's formula.

Next observe that $\tilde{J}_{i}=\left(h^{\tau_{i}}\right)_{*} J_{i}$, where $h^{\tau_{i}}: \pi^{-1}(p) \rightarrow \pi^{-1}\left(\tau_{i}(1)\right)$ is the diffeomorphism between the fibers naturally associated to the path $\tau_{i}$. This observation is a re-wording of [4, Prop. 1.1.1]. Further, by [4, Lem. 4.2], $h^{\tau_{i}}$ is a bi-Lipschitz map with bi-Lipschitz constant depending only on the length of $\tau_{i}$. It follows that $J_{i}$ is also a bounded vertical Jacobi field. 


\section{A SOUL WITH NON-COMPACT HOLONOMY}

In this section we construct a simply-connected nonnegatively curved open manifold such that the holonomy group of the normal bundle of the soul is non-compact.

Consider the following action of the Lie group $\mathbb{R}$ on $S^{2} \times \mathbb{C}^{2} \times \mathbb{R}$ :

$$
\left((\varphi, \theta), z_{1}, z_{2}, t_{0}\right) \stackrel{t}{\mapsto}\left((\varphi, \theta+t), e^{\pi i t} z_{1}, e^{\lambda \pi i t} z_{2}, t_{0}-t\right) .
$$

Here $(\varphi, \theta)$ denotes spherical coordinates on $S^{2}$, and $\lambda$ denotes an irrational real number. The quotient, $\left(S^{2} \times \mathbb{C}^{2}\right) \times \mathbb{R} \mathbb{R}=\left(S^{2} \times \mathbb{C}^{2} \times \mathbb{R}\right) / \mathbb{R}$, is diffeomorphic to $S^{2} \times \mathbb{C}^{2}$, and this identification provides a new nonnegatively curved metric $\hat{g}$ on $S^{2} \times \mathbb{C}^{2}$ under which the quotient map $S^{2} \times \mathbb{C}^{2} \times \mathbb{R} \rightarrow\left(S^{2} \times \mathbb{C}^{2}\right) \times \mathbb{R} \mathbb{R} \approx S^{2} \times \mathbb{C}^{2}$ becomes a Riemannian submersion.

Let $g$ denote the product metric on $S^{2} \times \mathbb{C}^{2}$, and let $V$ denote the Killing vector field associated to the $\mathbb{R}$ action on $S^{2} \times \mathbb{C}^{2}$; namely, $V\left((\varphi, \theta), z_{1}, z_{2}\right)=\left(\hat{\theta}, i z_{1}, \lambda i z_{2}\right)$. According to [1, Example 2], the new metric $\hat{g}$ on $S^{2} \times \mathbb{C}^{2}$ is obtained from $g$ simply by, at each point, rescaling the norms of vectors parallel to $V$ by a factor of $1 /\left(1+|V|^{2}\right)^{1 / 2}$.

It is easy to see that the soul of $M:=\left(S^{2} \times \mathbb{C}^{2}, \hat{g}\right)$ will still be the zero section, $\Sigma=S^{2} \times(0,0)$, and that the metric projection $\pi: M \rightarrow \Sigma$ will still be the projection $\left(q, z_{1}, z_{2}\right) \mapsto q$. It is straightforward to show that the horizontal distribution $\mathcal{H}$ of $\pi$ can be described as follows:

$$
\mathcal{H}_{\left((\varphi, \theta), z_{1}, z_{2}\right)}=\operatorname{span}\left\{(\hat{\varphi}, 0,0),\left(\hat{\theta}, \frac{|\hat{\theta}|^{2}}{1+|\hat{\theta}|^{2}} i z_{1}, \frac{|\hat{\theta}|^{2}}{1+|\hat{\theta}|^{2}} \lambda i z_{2}\right)\right\} .
$$

The two vectors in this expression correspond to the horizontal lifts of the spherical coordinate vectors $\hat{\varphi}$ and $\hat{\theta}$ in $T_{(\varphi, \theta)} S^{2}$.

If $\sigma(t)=(\varphi(t), \theta(t)), t \in[0,1]$, is a loop in $S^{2}$ based at $q:=(\varphi(0), \theta(0))$, it follows that the horizontal lift $\bar{\sigma}$ of $\sigma$ to the point $\left(q, z_{1}, z_{2}\right)$ in $M$ will end at the point $\bar{\sigma}(1)=\left(q, e^{\pi i t_{0}} z_{1}, e^{\lambda \pi i t_{0}} z_{2}\right)$ where $t_{0}=\int_{0}^{1} \frac{|\hat{\theta}|^{2}}{1+|\hat{\theta}|^{2}} \theta^{\prime}(t) d t$.

In particular, the set of points in $\pi^{-1}(q)$ which can be achieved as endpoints of horizontal lifts to $\left(q, z_{1}, z_{2}\right)$ of loops in $S^{2}$ at $q$ is exactly $\left\{\left(q, e^{\pi i t_{0}} z_{1}, e^{\lambda \pi i t_{0}} z_{2}\right) \mid t_{0} \in\right.$ $\mathbb{R}\}$. It follows from this that the holonomy group $\Phi$ of the normal bundle of $\Sigma$ is exactly:

$$
\Phi:=\left\{\left(\begin{array}{cc}
e^{\pi i t_{o}} & 0 \\
0 & e^{\lambda \pi i t_{0}}
\end{array}\right) \mid t_{0} \in \mathbb{R}\right\},
$$

which is isomorphic to $\mathbb{R}$.

\section{REFERENCES}

1. J. Cheeger, Some Examples of Manifolds of Nonnegative Curvature, J. Differential Geom. 8(1972), 623-628. MR 49:6085

2. J. Cheeger and D. Gromoll, On the structure of compete open manifolds of nonnegative curvature, Ann. of Math. 96 (1972), 413-443. MR 46:8121

3. J.H. Eschenburg, Comparison theorems and hypersurfaces, Manuscripta Math. 59 (1987), 295323. MR 89f: 53062

4. L. Guijarro and G. Walschap, The metric projection onto the soul, Trans. Amer. Math. Soc. (to appear). CMP 98:06

5. S. Kobayashi and K. Nomizu, Foundations of differential geometry I, Interscience Publishers (1963), J. Wiley and Sons. MR 27:2945 
6. G. Perelman, Proof of the soul conjecture of Cheeger and Gromoll, J. Differential Geom. 40(1994), 209-212. MR 95d:53037

7. V. Schroeder and M. Strake, Volume growth of open manifolds with nonnegative curvature, Ann. Global Anal. Geom., 8, no.2 (1990), 159-165. MR 92e:53052

8. M. Strake and G. Walschap, $\Sigma$-flat manifolds and Riemannian submersions, Manuscripta Math., 64(1989), 213-226. MR 90g:53054

9. J.W. Yim, Space of souls in a complete open manifold of nonnegative curvature, J. Differential Geom. 32 (1990), 429-455. MR 91j:53023

Department of Mathematics, University of Pennsylvania, Philadelphia, Pennsylvania 19104-6395

E-mail address: ktapp@math.upenn.edu 